\title{
NUEVOS MONUMENTOS FUNERARIOS DE BELORADO (BURGOS)
}

\author{
NEW FUNERARY MONUMENTS IN BELORADO (BURGOS)
}

\author{
MARTA FERNÁNDEZ CORRAL \\ Universidad del País Vasco/Euskal Herriko Unibertsitatea
}

\section{INTRODUCCIÓN ${ }^{1}$}

Presentamos a continuación cuatro nuevos hallazgos de inscripciones de época romana de Belorado (Burgos) que se suman a los ya conocidos de este municipio $^{2}$. En efecto, Belorado ha sido y sigue siendo un lugar prolífico en cuanto a hallazgos de estelas de época romana se refiere superando ya el número de 40 estelas funerarias a las que se les debe sumar las de la localidad vecina de Fresno de Río Tirón que, sin duda, forman parte del mismo conjunto.

Los monumentos funerarios han sido localizados en el propio casco urbano de Belorado o en sus inmediaciones, algunas veces reutilizados como material constructivo y otra abandonados o arrastrados por el río. Su presencia se pone en relación con el yacimiento de La Mesa, una pequeña meseta situada al Oeste de la villa, donde se han localizado abundantes restos arqueológicos de época romana. Los últimos trabajos arqueológicos realizados en 2014 han servido para constatar una primera fase alto-imperial (I-II d.C.) y una segunda fase más complicada de datar debido al arrasamiento del yacimiento pero probablemente vinculada a un momento más tardío del periodo imperial. Se localizó un área productiva con claros indicios de trabajo metalúrgico, textil y tal vez cerámico, confirmando, igualmente, que fue un asentamiento de cierta

1. Este trabajo se ha realizado dentro del Programa de Formación de Personal Investigador No Doctor del Gobierno Vasco y de las actividades efectuadas por el grupo de investigación del sistema universitario vasco IT-760-13. Agradecemos a los profesores $\mathrm{M}^{\mathrm{a}}$ Cruz González y Joaquín Gorrochategui su colaboración en la realización de la autopsia de las estelas así como las fotografías de las mismas al segundo. Igualmente, agradecemos a los informantes de esta contribución sus sugerencias para la mejora del texto original.

2. Fueron dados a conocer por el arqueólogo local Carlos Manero Sáez al Diario de Burgos que los publicó el día 17 de mayo de 2011 al que agradecemos sus indicaciones sobre estas inscripciones. entidad y organizado de forma urbana, con viviendas separadas por calles o vías ${ }^{3}$.

Varias de las estelas se encuentran en la actualidad desaparecidas y solo disponemos información sobre ellas a través publicaciones como la de Luís Ruiz de Huidobro (1950) y Olivia V. Reyes Hernando $(2000)^{4}$ que recogen la información de un manuscrito que Gerardo Saro Cano, notario de la villa, envió a la Comisión de Monumentos de Burgos. Sin embargo, entre las que conocemos más allá de la referencia a su texto, son en todos los casos, salvo uno $^{5}$, inscripciones realizadas en bloques fluviales de cuarcita sin trabajar, muy probablemente, seleccionados por su tamaño, forma y por tener una de sus caras los suficientemente lisa como para realizar una inscripción ${ }^{6}$.

Se caracterizan asimismo por estar exentos de decoración si bien en algunos de ellos se observan algunos signos o símbolos que comentaremos en la descripción de las piezas que trataremos a continuación.

Respecto a la estructura del texto y su contenido, las estelas de Belorado presentan en la mayoría de los casos el nombre del difunto seguido por su filiación y su edad. La mayoría de los difuntos poseen un

3. Agradecemos a los arqueólogos responsables del proyecto Joan Oller Guzmán, Diana de León Subías y Delia Eguiluz Maestro la información proporcionada al respecto (Oller, De León y Eguiluz, 2015).

4. A lo largo del artículo utilizaremos esta publicación como referencia para el resto de inscripciones de Belorado por tratarse del estudio más completo realizado hasta la fecha sobre el tema.

5. Se trata de una inscripción realizada sobre un soporte de caliza de forma discoidea decorado con abundantes motivos geométricos, fundamentalmente, circunferencias y motivos circulares de distinto tipo (Reyes Hernando, 2000, n²9).

6. Recuerdan al conjunto de estelas vadinienses en su forma exterior aunque no en su contenido (González Rodríguez, 1997) y a otras de Asturias, Soria y fuera de Hispania a las presentes en la IX Región de Italia (González Rodríguez, 2002, 95). 
nombre doble indígena aunque también encontramos ejemplos de duo y tria nomina así como la presencia de nombres latinos en la filiación. No contienen fórmulas introductorias como $D($ is $) M(\text { anibus })^{7}$ y la referencia al dedicante del monumento funerario es excepcional, sin embargo, la mención de la edad y la filiación del difunto es una constante, estando ausente en muy pocas ocasiones. Los monumentos funerarios que presentaremos a continuación se ajustan a las características generales que acabamos de señalar.

\section{ANÁLISIS DE LAS INSCRIPCIONES}

Figura 1. La primera de las inscripciones funerarias que presentamos fue localizada por el matrimonio Lecue-Osés en Redoña, Belorado (Burgos). Actualmente su lugar de conservación es la Oficina de Turismo de Belorado donde está expuesta al público ${ }^{8}$.

Se trata de un bloque fluvial de cuarcita de aspecto romboidal de grosor irregular en la parte superior e inferior, tal vez como consecuencia de una rotura posterior a la época en la que fue grabada. Sus medidas son [60] x50x20 cm. La parte superior izquierda de la pieza está fracturada y desaparecida y el campo epigráfico presenta algunos desconches. Debido a esta fractura faltan algunas letras del extremo izquierdo del campo epigráfico que hace que la interpretación de la inscripción se complique.

El campo epigráfico se encuentra situado en la parte superior del bloque, dividido en tres líneas que se adaptan a las medidas de la pieza que guardan un interlineado apenas perceptible. Si bien la fractura superior izquierda impide asegurarlo totalmente, parece que la última línea correspondiente a la edad del difunto estaría centrada respecto al resto, un rasgo habitual en las estelas de este conjunto.

En lo que se refiere a la escritura del texto, las letras miden entre 3,5 y $4 \mathrm{~cm}$ y no contamos con nexos $\mathrm{o}$ interpunciones entre las distintas palabras. Las letras son de factura algo irregular que tiende a alargarse y gran parte de ellas tienen ápices si bien no es igual de evidente en todas. En cuanto a las características paleográficas que más llaman la atención, debemos señalar la presencia de A sin travesaño, trazado de la $\mathrm{B}$ estrecha mientras que la $\mathrm{M}$ es abierta y la diferencia entre el contorno anguloso de la letra $\mathrm{S}$ de la línea 1 que es algo más redondo en la línea 2.

7. A excepción de la estela dedicada a Quemia Maglaena (Reyes Hernando, 2000, $\mathrm{n}^{\circ} 1$ ) en la que encontramos la fórmula $H S E$ o la dedicación a los Dioses Manes en la estela discoidea dedicada a Aurelio Materno indicada en la nota 5.

8. Agradecemos a Conchi García Rodríguez, responsable de la Oficina de Turismo de Belorado, las facilidades dadas para su estudio.

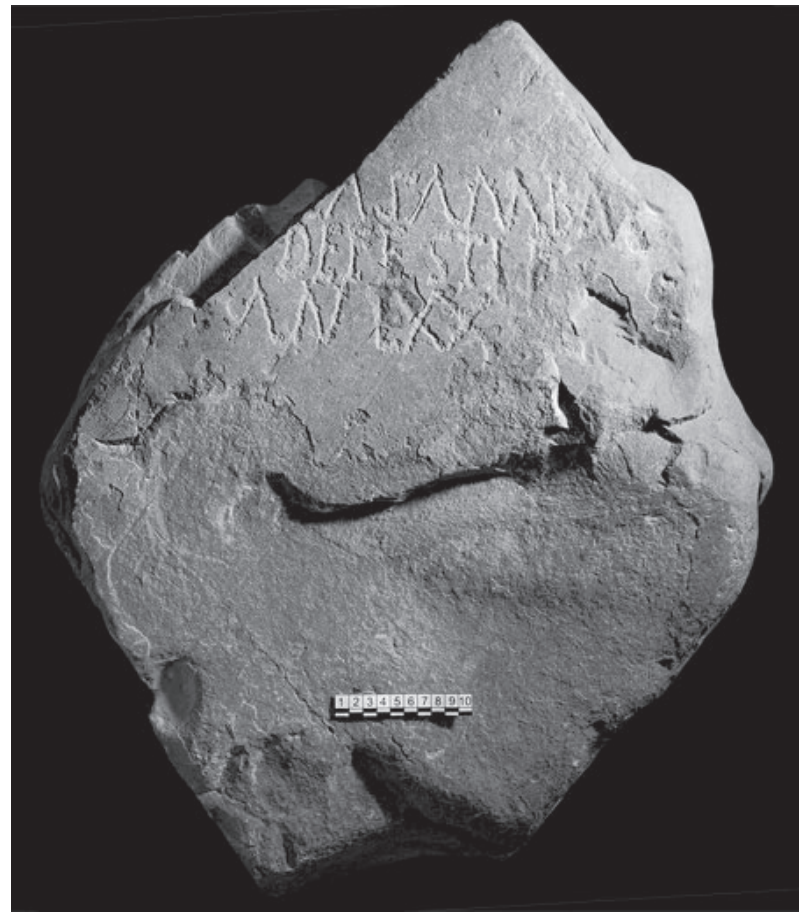

Figura 1: Estela funeraria dedicada a Casia Ambada.

Transcripción:

$+A S A M B A$

DEFESTI F

AN LXX

Interpretación:

[C]as(siae) Amba /d<a>e Festi f(iliae) / an(norum) $L X X$

Traducción:

Para Cassia Ambada, hija de Festo, (fallecida a la edad) de 70 años.

El monumento funerario carece de decoración y, al igual que en las siguientes inscripciones, no contamos con ningún elemento que nos permita conocer su cronología. El análisis del contenido del texto nos muestra una inscripción que sigue los elementos propios de las inscripciones de este conjunto anteriormente mencionadas, es decir, nombre del difunto + filiación + edad del fallecido.

En cuanto a la onomástica, como ya hemos mencionado, la fractura de la parte superior izquierda de la pieza hace que sea complicado determinar cuál es el nombre del difunto completo. Creemos que la opción más factible es que uno de sus nombres sea $A m b a d<a>e$, que ha sido separado entre las líneas 1 y 2 para adaptarse a las medidas y condiciones del irregular bloque fluvial. Conocemos paralelos de este nombre en el cercano lugar de Villafranca de los Montes de Oca (Burgos) Valeria[e] Ambadae lib. (CIL II 2909) y Corneliae Ambadae (CIL II 2908) así como en León Ambadus Palari (CIL II, 5709) y puede que en Mata de la Hoz (Cantabria) Ambad(us) Al(ius) 
Negali f. (ERCan, $\left.\mathrm{n}^{\circ} 6\right)$, si bien este último paralelo no es tan claro porque se trata de la interpretación de una abreviatura.

Aceptando la presencia del nombre Ambade, quedaría por resolver a qué corresponden las primeras letras de la línea 1 en la que podemos leer las letras AS precedidas por un trazo horizontal bajo que, por sus características, podría tratarse de una C, E, G, L, P, Z9 . Teniendo en cuenta esto, hemos tratado de buscar paralelos onomásticos que nos proporcione opciones de interpretación respecto a cuál debería ser el desarrollo del nombre del difunto.

La búsqueda de nombres terminados en -AS ha dado como resultado numerosos antropónimos, sin embargo, ninguno de los nombres parece mostrar una opción convincente, siendo la mayoría de origen heleno. Debemos recordar que la terminación -AS no es algo común entre los nombres latinos o los nombres indígenas hispanos por lo que era de esperar que la mayoría de los paralelos encontrados fuesen antropónimos de ámbito heleno y concentrados sobre todo en Roma y la zona de Campania. El único ejemplo en Hispania es el de Helias (CIL II, 3023) que, sin duda, se trata de un nombre de origen griego.

Por otro lado, entre los escasos resultados de la búsqueda de posibles abreviaturas de nombres en -AS, hemos encontrado lo que pensamos que podría ser la interpretación más factible. Nos referimos al nomen latino Cassius, $-a$, que aparece abreviado como Cas(sius, $-a$ ) en numerosas ocasiones y del que tenemos otro ejemplo en un lugar cercano a Belorado, en Barcina de los Montes (AE 1976, 292) donde el nomen aparece en una dedicación votiva al dios indígena Vurouius por parte de un individuo con tria nomina llamado Lucius Cassius Flaccus.

De este modo, Cas(sia) Ambada portaría un nombre mixto, formado por un nomen latino seguido de un nombre indígena que podríamos considerar una variante de Ambatus, - $a$, el antropónimo indígena que más se repite en la Península Ibérica ${ }^{10}$ y del que tenemos varios paralelos en Belorado mismo (Reyes Hernando, 2000, $\mathrm{n}^{\circ}$ 5, 9, 18, 20, 21, 24, 25).

Finalmente, el nombre de su padre sería Festus, un antropónimo latino de sobra conocido del que, solo por mencionar alguno cercano, tenemos paralelos en Lara de los Infantes (Burgos) Sempronio Festo Lougei $f .($ ERLara, 115$)$ y el que seguramente es una variante

9. Debido que la técnica de repiqueteado utilizada para inscribir el texto hace en ocasiones que las letras sean irregulares, hemos realizado búsqueda de paralelos suponiendo que el resto de trazo podría pertenecer también a un ápice de las letras F, I, P, Q, T o X. Sin embargo, no hemos obtenido ningún resultado que pueda encajar en nuestro epígrafe.

10. Ambatus, $-a$ es el nombre indígena más veces constatado en la Península Ibérica y ocupa el puesto decimotercero en la frecuencia de antropónimos (Abascal Palazón, 1994, 260). del mismo nombre en La Puebla de Arganzón (Burgos) Lucia Fesetina (ERAlavesa, 78) ${ }^{11}$.

La avanzada edad de la difunta no desentona con el resto de edades señaladas en muchas de las inscripciones de Belorado. Sin embargo, se debe tener en cuenta que se ha observado que la exactitud de la edad expresada tiende a redondearse y a exagerarse cuanto mayor es, por lo que no debe ser considerada un dato demográfico fehaciente ${ }^{12}$.

Figura 2. La segunda de las inscripciones es también un monumento funerario localizado en una finca próxima al yacimiento de La Mesa que hemos mencionado previamente. Se conserva y expone junto con las restantes en la Oficina de Turismo de Belorado.

Lo primero que llama la atención de este epitafio son sus grandes medidas, [96] x86x 15,5 cm que lo convierten en una gran laja fluvial de cuarcita de forma casi cuadrangular. Así, esta pieza destaca sobre el resto debido a su gran tamaño y a que es bastante menos gruesa que el resto de bloques del conjunto. Su conservación es buena exceptuando la parte superior central derecha que se encuentra desconchada y algunas fracturas en la parte inferior. Sin embargo, esto no afecta al campo epigráfico que se encuentra situado en la parte superior del bloque distribuido en tres líneas -excluyendo los símbolos de la parte superior- que mide $25 \times 62 \mathrm{~cm}$. Las dos primeras líneas del texto guardan margen a la izquierda y la última, correspondiente a la edad, se encuentra centrada respecto a las superiores.

Las letras miden cerca de $4,5 \mathrm{~cm}$ y ninguna de ellas forma nexos. El espacio de interlineado es mínimo siendo casi inexistente entre las primeras líneas y de medio centímetro entre las líneas 3 y 4 . Contamos con interpunciones en la segunda línea del texto, entre el nombre del padre y la abreviatura $f($ ilia), y la referencia a la edad anno(rum), sin embargo, no se aprecia ninguna en la primera línea para separar los dos elementos del nombre de la difunta. Los signos de interpunción son ligeramente alargados, producto seguramente de un solo golpe de cincel. Respecto a los rasgos paleográficos, es de destacar el uso de A con travesaño oblicuo que parte del trazo derecho de la letra, la $\mathrm{G}$ abierta con pedúnculo recto y el trazado abierto de $\mathrm{M}$ y $\mathrm{N}$.

11. Esta interpretación ha sido realizada tras apartar la opción de que toda la línea 2 estuviese dedicada al nombre del padre llamado Defestus, que se trataría de un hápax. Al existir paralelos para el antropónimo Ambadus, $-a$, preferimos no ser responsables de la inclusión ficticia de un nuevo nombre en el repertorio onomástico y decantarnos por la interpretación ofrecida en el texto. Habría que estar atentos, sin embargo, a la aparición de nuevos antropónimos que se pudiesen relacionar con Defestius, ya que elementos de la ordinatio de la inscripción hacen pensar que es factible que se pudiese interpretar como [C]as(sio, -ae?) Amba[to, ae] Defestif(ilio, $-a e)$.

12. Ver notas 21 y 22. 


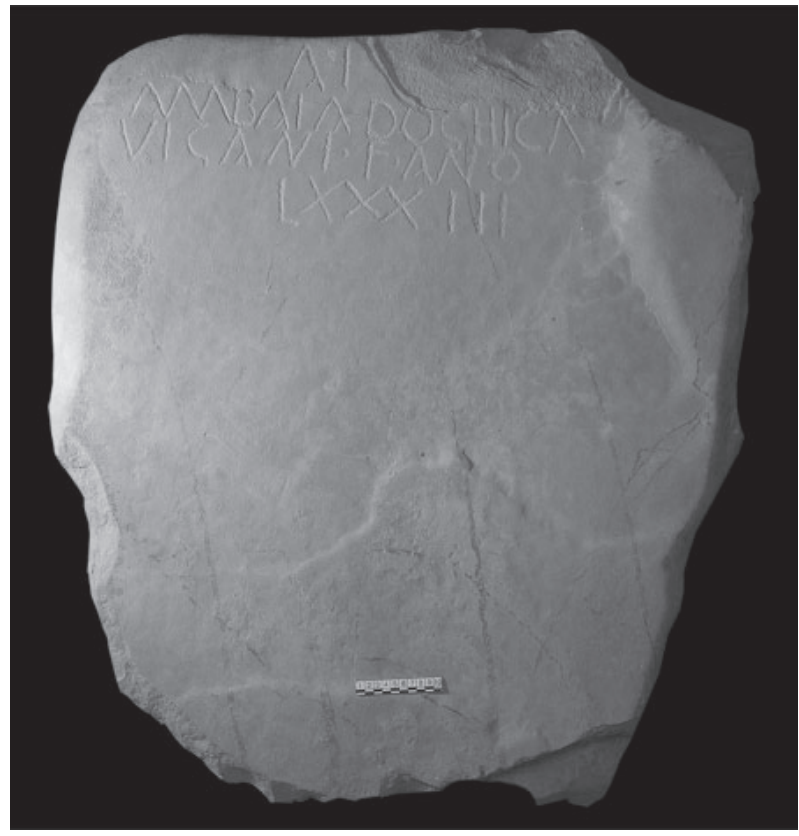

Figura 2: Estela funeraria dedicada a Ambaia Doghica.

Transcripción:

AMBAIA DOGHICA

$V I G A N I \cdot F \cdot A N O$

LXXXIII

Interpretación:

Ambaia Doghica / Vigani $\cdot f($ ilia $) \cdot a n<n>o($ rum $)$ /

LXXXIII

Traducción:

Ambaia Doghica, hija de Vigano, (fallecida a la edad) de 83 años.

En la parte superior central de la pieza encontramos unas marcas que podrían ser consideradas letras o símbolos de interpretación desconocida. La forma de estos dos elementos recuerda a una letra A con travesaño oblicuo que partiría del trazo derecho y una línea vertical que podría ser identificada con la letra I, ambas miden $6 \mathrm{~cm}$ de alto. Sean letras, símbolos o algún tipo de decoración, no conocemos cuál es su significado o interpretación. Sin embargo, no creemos que se trate parte del texto sino que debe ser puesto en relación con el resto de signos que aparecen en al menos 10 inscripciones más del conjunto de Belorado en las que también aparecen estos elementos de interpretación complicada ${ }^{13}$.

13. Contamos con estas marcas en las $\mathrm{n}^{\mathrm{o}} 1,3,4,7,9$ y 13, y posiblemente también en las número $\mathrm{n}^{\circ} 11$ y 20 , de la publicación de Reyes Hernando (2000) así como en la nº de Fernández Corral y Carcedo de Andres (2015), en las n ${ }^{\circ} 1$, 4 y 5 de Gorrochategui y Fernández Corral (2015) y en las $\mathrm{n}^{\mathrm{o}} 2$ y 4 del presente trabajo.
El epitafio carece de elementos que nos permitan conocer su cronología ya que se repite una vez más el esquema habitual de los monumentos funerarios del lugar: nombre del difunto + filiación + edad del fallecido. Un elemento a destacar es la utilización de la abreviatura ano para annorum en lugar del habitual an que encontramos en la mayoría de inscripciones del conjunto.

Respecto a la onomástica, como es habitual, contamos con el nombre de, en este caso, la difunta $\mathrm{Am}$ baia Doghica y su filiación paterna Viganus. El primer elemento de la nomenclatura de la difunta no cuenta más que con cuatro ejemplos situados en la provincia limítrofe de Araba/Álava si bien se trata de su forma masculina o derivados. Nos referimos a las inscripciones en las que aparece Munatius Fuscus Ambaici f(ilius) (CIL II, 2935; ERAlavesa 45), Ambaius Sermii f(ilius) (CIL II, 2951; ERAlavesa 24), A[m]baicus [A] mbani f(ilius) (HEp 01, 12) y finalmente [---]cus Ambai f(ilius) (ERAlavesa 16). La primera fue localizada en el yacimiento de Iruña-Veleia, las dos siguientes en Contrasta y la última en San Román de San Millán. Nos gustaría recalcar que se trata claramente de $\mathrm{Am}$ baia y no Ambata ya que no hay resto de ningún rasgo horizontal sobre la letra I.

El segundo elemento del nombre de la difunta, Doghica, no tiene ningún paralelo onomástico y se trata, por lo tanto, de un hápax. Podría tratarse de un nombre formado con el sufijo -icus, -a pero la raíz del mismo nos sigue siendo desconocida.

Por último, el nombre del padre de la difunta es de sobra conocido tanto en su versión sorda (Vicanus) como sonora, en este caso (Vallejo, 2005, 449-450). Tenemos ejemplos en Hispania pero también en Brittania (RIB I, 111), en las Galias (CIL XII, 4804) e incluso en el norte de África (CIL VIII, 4910). Como paralelos más cercanos, hay otro epígrafe de Belorado donde se menciona a una difunta llamada Iulia Viganica (Reyes Hernando, 2000, $n^{\circ}$ 19) así como el epígrafe del que presentamos la edición seguidamente.

Como en el caso anterior, la difunta presenta una edad muy avanzada que pensamos que se trata de una exageración, tal y como suele suceder entre los difuntos que alcanzan cierta edad ${ }^{14}$.

Figura 3. La siguiente inscripción fue localizada en el término de El Solo (Belorado) por F. Rioja. Se conserva y expone junto con las restantes en la Oficina de Turismo de Belorado. El soporte de la inscripción es un bloque de cuarcita de forma trapezoidal colocado con su parte más ancha hacia arriba y cuyas medidas son $30 \times 38 \times 22 \mathrm{~cm}$. Se encuentra algo erosionado pero esta circunstancia no dificulta demasiado la lectura.

El campo epigráfico se adapta a la parte superior del bloque fluvial sin guardar margen alguno y se

14. Ver notas 21 y 22 si bien en este caso la edad no ha sido redondeada. 
distribuye en 3 líneas guardando un interlineado de entre 0,5 y $1 \mathrm{~cm}$. La última de ellas, correspondiente a la edad de la difunta, está centrada como en los casos anteriores.

En cuanto a la escritura, la medida de las letras oscila entre 3 y $3,5 \mathrm{~cm}$ en las dos primeras líneas y 3,5 y $4 \mathrm{~cm}$ en la tercera. Se observa una interpunción de forma redonda entre cada una de las palabras y el nexo $A E$ en la línea 2. Los rasgos paleográficos más reseñables son la tendencia a alargarse de la letras, el uso combinado de A de travesaño horizontal (línea 2), A de travesaño oblicuo que parte de la izquierda (línea 2) y de la derecha (línea 1) y sin travesaño formando parte de un nexo $A E$ (línea 2), O muy redonda, G con pedúnculo recto hacia arriba y $\mathrm{V}$ muy abierta.

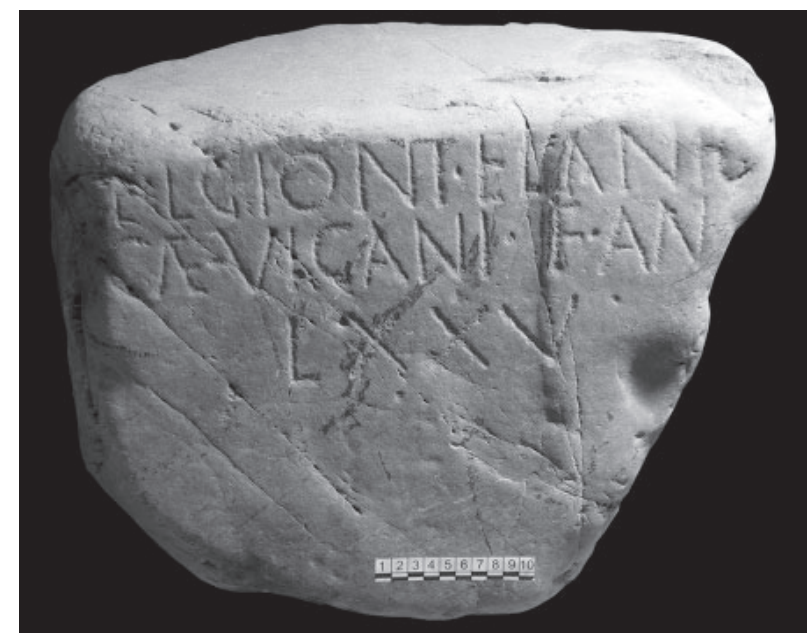

Figura 3: Estela funeraria dedicada a Elgio Elanica.

Transcripción:

ELGIONI $\cdot$ ELANIC

$C A E \cdot V I G A N I \cdot F A N$ $L X X V$

Interpretación:

Elgioni $\cdot$ Elanic/cae $\cdot$ Vigani $\cdot$ f(iliae) an(norum) / LXXV

\section{Traducción:}

Para Elgio Elanica, hija de Vigano, (fallecida a la edad) de 75 años.

El monumento funerario carece de decoración y no contamos con ningún elemento que nos permita conocer la cronología de la inscripción ya que, como las anteriores, el texto no nos da información al respecto. Una vez más, el texto está formado por el nombre del difunto + filiación + edad del fallecido.

En cuanto a la onomástica, como en el caso anterior, contamos con el nombre de la difunta y su filiación. La nomenclatura de la difunta está formada por dos nombres indígenas declinados en dativo de los que conocemos paralelos. Del primero de ellos, Elgio$n i$, conocemos un único paralelo de una inscripción también localizada en Belorado Elgion(i) Alebiae (Gorrochategui Churruca y Fernández Corral, 2015). Si bien este sería el único paralelo, conocemos antropónimos que contienen la raíz ELG-/ELC- en lugares como Ávila donde hay testimonio de un Elciamo Sangen... (ERAv 42), en Hinojosa de Jarque (Teruel) [--] Proculus [--] Elguanus (ERTer 14), en Villalcampo (Zamora) Elguenif. (HEp 7, 1085) o Elcuius Modestinus Emeritensis en Rosinos de Vidriales (Zamora) (HEp 6, 996).

Respecto al segundo elemento del nombre de la difunta, se trata de un antropónimo seguido del sufijo -icca que podría estar haciendo referencia a su cognatio o grupo suprafamiliar. Sin embargo, teniendo en cuenta el resto de inscripciones de Belorado en las que lo más común es que el difunto tenga dos nombres, creemos que Elanicca se trata de un segundo elemento del nombre y no una expresión directa de un grupo suprafamiliar ${ }^{15}$.

Conocemos paralelos tanto del que sería el nombre de origen Elanus (CIL II, 5819 y 5716 o ERA 186, nº0-f) como de Elanicus, - a (EBrag 27 y AE 1981, 544), sobre todo en el área cántabra y sus inmediaciones. Igualmente, también se ha registrado la forma acabada en -cum en Yecla de Yeltes (Salamanca) Caenia Lupi f. Elanic(um) (CIL II 5034 y González Rodríguez, 1986, 68 n¹19) y en Lara de los Infantes (Burgos) Arcea Elanioca Paterni, f. (ERLara, 146).

Finalmente, el nombre del padre de la difunta coincide con el del epitafio anterior y, como ya hemos dicho, es conocido tanto en su variante sorda como sonora y tenemos ejemplos tanto Hispania como en otras provincias como Brittania, las Galias y el norte de África. Los paralelos más cercanos son los mencionados en la inscripción anterior.

Figura 4. Por último presentamos la edición de un epitafio localizado en el cauce del río Verdeancho por $\mathrm{S}$. Escolar que se conserva y expone junto con las restantes en la Oficina de Turismo de Belorado. El monumento epigráfico es un bloque de cuarcita de forma trapezoidal, algo más irregular que la anterior y con unas medidas algo superiores: $62 \times 62 \times 26$. A pesar de que ha sufrido algún pequeño desconchamiento en la parte superior izquierda que afecta a la primera letra de la línea 3 , se puede decir que la conservación de la pieza en general es buena.

El texto está situado en la parte superior del bloque que es además, la parte más lisa y regular de la pieza. La inscripción está dividida en cuatro líneas que se adaptan al relieve del bloque y guardan un interlineado de entre 0,5 y $1 \mathrm{~cm}$, siendo algo mayor entre las dos últimas líneas. Se aprecia, como en ocasiones anteriores,

15. Sobre los grupos suprafamiliares o cognationes del área indoeuropea de Hispania ver González Rodríguez y Ramírez Sánchez, 2011; González Rodríguez, 1986, 1994; Ramírez Sánchez, 1999, 2003, 2004, 2007. 
un intento de centrar la última línea correspondiente a la edad del difunto.

Sobre la escritura, la medida de las letras oscila entre 5,5 y $7 \mathrm{~cm}$ en la primera línea y 5,5 y $6 \mathrm{~cm}$ en la segunda y la tercera. No hay nexos y en lo referente a interpunciones, solo se aprecia un pequeño rasgo curvo en la última línea entre an(norum) y la edad del difunto. Las letras son regulares, con forma tendente a la cuadratura en ocasiones. Se combina el uso de A sin travesaño (línea 1) y A con travesaño oblicuo que parte desde el trazo derecho (línea 4), la $\mathrm{O}$ es muy redonda, la $\mathrm{G}$ tiene un pedúnculo vertical; el contorno de la $\mathrm{S}$ es redondeado y la $\mathrm{R}$ es la única letra que presenta ápices de forma clara. La letra $\mathrm{E}$ es algo irregular ya que mientras que sus trazos horizontales son cortos en la mayoría de los casos, se alargan en la palabra Hortens.

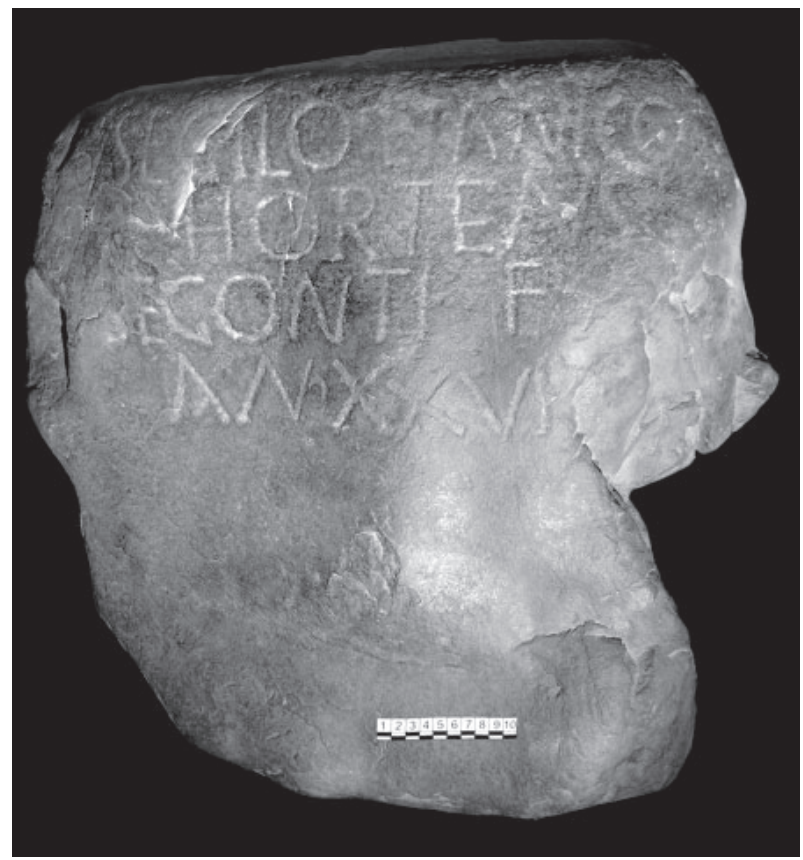

Figura 4: Estela funeraria dedicada a Segilo Etanico.

Transcripción:

SEGILO ETANICO

HORTENS

SEGONTI F

$A N \cdot X X V I$

Interpretación:

Segilo Etanico / Hortens(i?) / Segonti f(ilio) /

an(norum) XXVI

Traducción:

Para Segilo Etanico, hijo de Hortensio Segontio, (fallecido a la edad) de 26 años.

Al igual que en el epitafio dedicado a Ambaia Doghi$c a$, este también presenta signos de interpretación desconocida. El primero de ellos es una circunferencia de $4,5 \mathrm{~cm}$ de diámetro dibujada sobre la línea 1 , concretamente, sobre la letra I de Etanico. Asimismo, también encontramos un símbolo en la cara superior del bloque que consiste en un círculo de $6,5 \mathrm{~cm}$ de diámetro del que sale del centro del lado derecho una línea horizontal de $5,5 \mathrm{~cm}$ a la que, a su vez, se le adosa perpendicularmente una tercera línea de $8 \mathrm{~cm}$ (Fig. 5). Como en otras ocasiones, no sabemos si estas marcas tienen algún significado simbólico o se trata de motivos decorativos ${ }^{16}$.

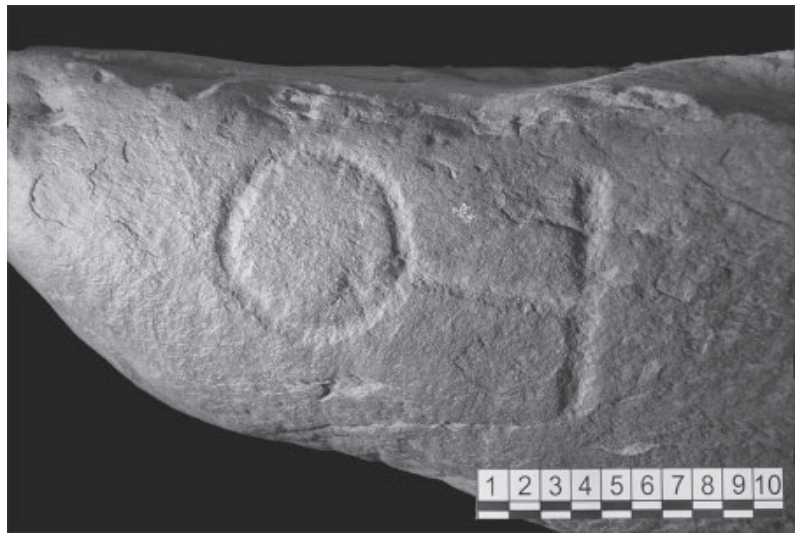

Figura 5: Detalle de la marca de la estela funeraria dedicada a Segilo Etanico.

Una vez más, la ausencia de fórmulas funerarias estandarizadas $\mathrm{u}$ otro tipo de indicios, nos impide proponer la cronología de la inscripción aunque el esquema de la misma varía respecto a las anteriores ya que aparece un antropónimo más que los que podríamos esperar: Hortens. El papel que jugaría el nombre Hortens no está claro ya que, al estar abreviado, no conocemos cuál sería su declinación y, por lo tanto, a quién corresponde el nombre. Existen tres posibilidades en cuanto a la declinación con la que habría que completarlo:

- Dativo Hortens (io) y como consecuencia, un tercer elemento del nombre del difunto.

- Nominativo Hortens(ius) y, por lo tanto, el nombre del dedicante de la inscripción.

- Genitivo Hortens (i) presentándose como el primer nombre del padre, es decir, formando parte de la filiación.

Nos parece que la opción más plausible podría ser la tercera, es decir, que la filiación del padre esté indicada con un nombre doble que comprende un conocido nomen latino seguido de un cognomen indígena. $\mathrm{Si}$ bien no es habitual que en la filiación se indique más de un nombre, contamos con varios ejemplos de ello en Lara de los Infantes (Burgos): Coemeae Dessicae Visadi Aquini f. (ERLara 158), Arcea [...]uca Ambati Terenti f. (ERLara 160), Elaesus Petolus Lougei Petraioci fil. (ERLara 70), Visado Presso Elaesi Pulliani

16. Ver nota 13 
f. (ERLara 72), así como en Contrasta (Araba/Álava) Segontius Ambati Vectif. (ERAlavesa 28).

En contra de la primera opción juega el hecho de que Hortensius en un nomen latino que no deberíamos esperar encontrar en tercera posición de una nomenclatura, a no ser, que el segundo elemento del nombre, Etanico, no solo tenga como origen el nombre de un grupo suprafamiliar sino que efectivamente, se nos este indicando que pertenece a él. Sin embargo, nos parece que sería extraño igualmente que viniese indicada entre los dos nombres del difunto y no tras el nombre completo y su filiación, como es más habitual ${ }^{17}$. La segunda opción ha sido igualmente descartada porque también resultaría demasiado extraña la presencia del dedicante del monumento funerario situado entre el nombre del difunto y su filiación.

Partiendo de la hipótesis anterior, el análisis onomástico permite constatar la presencia de dos individuos con antropónimos formados por dos elementos: Segilus Etanicus y Hortens(ius) Segontius. Tanto Segilus como Segontius son nombre indígenas bien conocidos en la Península ibérica creados a partir del radical $\operatorname{seg}^{\mathrm{h}}$ - «victoria» del que también se han creado topónimos como Segobriga, Segisama, Segisamo, etc. (Vallejo, 2005 397). De Segilus, $-a$ conocemos paralelos en Tarragona L. Baebiu[s] Segilu[s] y $[L$. Baebi L(uci) l. [Se]gilus (CIL II, 4338 y 6118 respectivamente), en Laguardia (Araba/Álava) Segilus (HAE 2546), en Botorrita (Zaragoza) Segilus Annicum Lubbi $f$. (HEp 3, 415) y en Belorado mismo Segile Maglene, Segil[o] Elarco[--], Segilo Aespanco [A]mbata $<e>$ $f$., [A]mbato Burgae Segili f. (Reyes Hernando, 2000, $\mathrm{n}^{\mathrm{o}} 8^{18}, 14,20$ y 21 respectivamente).

Los paralelos de Segontius son más numerosos concentrándose sobre todo en Navarra y Araba/Álava con extensiones hacia La Rioja, Burgos y el territorio astur (Vallejo, 2005, 397). En Belorado mismo tenemos el ejemplo de [Am]batae [Se]gon [ti]e (Gorrochategui Churruca y Fernández Corral, 2015).

Respecto a Etanico, no contamos con ningún paralelo. Creemos que se trataría de un nombre formado por un antropónimo indígena, Etanus, $-a$, del que no conocemos paralelos seguido del sufijo + icus, $-\mathrm{a}$, relacionado con la designación de grupos suprafamiliares. Sin embargo, como ya hemos adelantado, no creemos que, por su posición en el texto, se esté haciendo referencia a su cognatio sino que se trataría de una forma que ha pasado al repertorio antroponímico al igual que Elanicca, en la inscripción que acabamos de editar con el número 3.

Hortensius, $-a$ es un nomen latino del que conocemos algunos ejemplos en Hispania: Lugo (HEp 10 362), Ampurias (HEp 12 159), Segobriga ( $A E$ 2011, 578) y Osuna (HEp 4 825=AE 1991, 1020). Sin embargo, está bien atestiguado en el resto de provincias

17. Ver nota 15

18. Propone la lectura Segil $<a>[e] /$ Elarco/[r]um? [..] / [--]. romanas, especialmente y como era de esperar, en Italia, pero también hay numerosos ejemplos en Africa Proconsularis y Galia Narbonensis.

$\mathrm{Al}$ igual que en la primera y segunda inscripción, la edad del difunto resulta demasiado elevada y no se debe tomar como un dato objetivo ${ }^{19}$. Trataremos este tema en las conclusiones del artículo.

\section{CONCLUSIÓN}

En resumen, estos cuatro epígrafes localizados en Belorado nos permiten conocer mejor la población de época romana de este territorio y acercarnos más a las prácticas funerarias y epigráficas de la zona. Como ya hemos adelantado en la introducción, los monumentos funerarios analizados se adaptan a las características generales del lugar aunque debemos mencionar algunos elementos que destacan entre ellos como el gran tamaño del número 2 o la filiación expresada con un nombre doble en el número 4.

Resulta imposible especificar la cronología de las inscripciones aquí presentadas por haber sido localizadas fuera de contexto arqueológico y la ausencia de formularios $\mathrm{u}$ otros elementos que nos permitan determinarla. En cualquier caso, podríamos pensar que su producción se realizó entre los siglos I-III d. C., es decir, en el momento en el que podemos situar la mayoría de monumentos epigráficos de estas características en el Imperio romano.

En lo que se refiere a la onomástica, tres de los difuntos presentan un nombre doble indígena mientras que el cuarto, creemos que podría tratarse de un duo nomina compuesto por un nomen latino y un cognomen indígena al que le sigue la filiación, presente en los cuatro casos. La filiación es paterna y está señalada con un único nombre seguido por la abreviatura f(ilio, ae) excepto, como ya hemos dicho, en la inscripción $n^{\circ} 4$.

Entre los difuntos, encontramos dos mujeres y dos hombres. Esta paridad sigue la llamativa tendencia de los epígrafes de Belorado en los que el número de mujeres representadas es muy elevado, alcanzando casi la misma cifra que las dedicadas a hombres ${ }^{20}$. Si bien la presencia notable de mujeres en los epígrafes de Hispania ya fue señalada por Saller y Shaw $(1984,138)$, su presencia se acusa sobre todo como dedicantes de monumentos funerarios y no como difuntas.

Llama igualmente la atención la edad elevada que presentan tres de los cuatro individuos: 70,75 y 83 años, una edad que supondría una esperanza de vida excesivamente alta respecto a lo que se supone para

19. Ver nota 21 y 22.

20. Entre las inscripciones localizadas en Belorado que pueden ser claramente adscritas a uno de los dos sexos identificamos 19 dedicadas a hombres y 16 a mujeres. 
esta cronología ${ }^{21}$. Estos epitafios no son un caso aislado ya que, como ya hemos mencionado anteriormente, hay una tendencia generalizada a exagerar y redondear las edades de los difuntos ${ }^{22}$. Se ha propuesto que este fenómeno estuviese relacionado, por un lado, con la falta de un registro o control de la edad de cada individuo que provocaría dudas sobre su cifra exacta cuanto más alta fuera esta, provocando así el redondeo de su edad y, por otro, con un reconocimiento social de los miembros de edad más avanzada que hiciera positiva la exageración de la misma. Esto, junto al hecho de que la representación de los difuntos responde a razones sociales y culturales, hace que se hayan descartado las edades que aparecen en los epitafios como datos demográficos objetivos ${ }^{23}$.

Por otro lado, dos de las cuatro piezas presentadas tienen signos situados en la parte superior del texto cuyo significado resulta difícil de esclarecer. Como ya hemos adelantado en la introducción, estas marcas aparecen en varias inscripciones del conjunto de Belora$\mathrm{do}^{24}$ y parecen propias del mismo, ya que no conocemos paralelos entre otros conjuntos epigráficos. Lamentablemente, no hemos conseguido establecer ninguna relación entre su presencia o ausencia y las personas representadas en los epitafios, es decir, no parecen estar vinculados, por ejemplo, a una variante de sexo, edad $\mathrm{u}$ onomástica del difunto. Por lo tanto, la definición de su función como marcas, símbolos o elementos decorativos queda sin explicación por el momento.

Finalmente, nos gustaría concluir diciendo que a pesar del aspecto «tosco» o poco cuidado de estos monumentos funerarios, el análisis del texto nos muestra elementos que siguen claramente los estándares del uso de la epigrafía o escritura monumental de época romana: abreviaturas comunes como f(ilius, a) o an(norum), nexos de letras y utilización de interpunciones. Creemos que el uso de este tipo de soportes es una elección consciente de los habitantes de este lugar que podría responder a razones sociales, culturales, religiosas... y no a una limitación material o técnica, ya que encontramos monumentos funerarios que adecuan a las características estándar de los soportes de esta época a escasos kilómetros de Belorado ${ }^{25}$.

21. Contamos con otros ejemplos de individuos que alcanzan o superan los 70 años entre otras inscripciones de Belorado (Reyes Hernando, 2000, $\mathrm{n}^{\circ}$ 1, 3, 14, 15, 19, 20, 22, 23 y 25) aunque el redondeo de las cifras no siempre está presente.

22. Un caso paradigmático sería el del Norte de África donde se observa una abundante presencia de hombres de avanzada edad (Maurin y Khanoussi, 2002, 84-90).

23. Sobre la tendencia a redondear y exagerar la edad de los difunto en época romana ver Duncan-Jones (1990, 80-92) y el trabajo de Scheidel (2001, 17-19) junto con la bibliografía ahí citada.

24. Ver nota 13.

25. Sin embargo, a menos de 50 kilómetros, en Poza de la Sal y sus alrededores encontramos otro soporte de características particulares como son las estelas en forma de casa

\author{
Marta Fernández Corral \\ Dpto. Estudios Clásicos \\ Edificio Flandes \\ C/ Tomás y Valiente, $\mathrm{s} / \mathrm{n}$ \\ Universidad del País Vasco/ \\ Euskal Herriko Unibertsitatea \\ 01006 Vitoria - Gasteiz \\ mfernandezcorral@gmail.com
}

\section{BIBLIOGRAFÍA}

ABASCAL PALAZÓN, J. M., 1994: Los nombres personales en las inscripciones latinas de Hispania, Murcia.

ABASCAL PALAZÓN, J. M., 2014: «Oficinas y estilos en el hábito epigráfico de la Hispania romana», en M. Bustamante Álvarez y D. Bernal Casasola (eds.), Artífices Idóneos. Artesanos, talleres y manufacturas en Hispania, 143-168, Mérida.

ABÁSOlO ÁlVAREZ, J. A., ALBERTOS FIRMAT, M. L. y ELORZA GUINEA, J. C., 1975: Los monumentos funerarios de época romana, en forma de casa, de la región de Poza de la Sal (Bureba, Burgos), Burgos.

DUNCAN-JONES, R., 1990: Structure \& Scale in the Roman Economy, Cambridge.

EBrag $=$ REDENTOR, A., 2002: Epigrafia romana da região de Bragança, Lisboa.

EDMONDSON, J., 2015: «Inscribing Roman Texts. Officinae, Layout and Carving Techniques», en C. Bruun y J. Edmondson, The Oxford Handbook of Roman Epigraphy, 111-130, Oxford.

$E R A=$ DIEGO SANTOS, F., 1985: Epigrafia romana de Asturias, Oviedo.

ERAlavesa = ELORZA GUINEA, J. C., 1967: «Ensayo topográfico de epigrafía romana alavesa», Estudios de Arqueología Alavesa, 2, 119-186.

$E R A v=$ HERNANDO SOBRINO, M. R., 2005: Epigrafía Romana de Ávila, Bordeaux-Madrid.

ERCan = IGLESIAS GIL, J. M. y RUIZ GUTIÉRREZ, A., 1998: Epigrafía Romana de Cantabria. Bordeaux-Santander.

ERLara = ABÁSOLO ÁLVAREZ, J. A., 1994: Epigrafía romana de la región de Lara de los Infantes, Burgos.

ERTer $=$ NAVARRO CABALLERO, M., 1994: La epigrafía romana de Teruel, Teruel.

FERNÁNDEZ CORRAL, M. y CARCEDO DE ANDRES, B. P., 2015: «Dos nuevas estelas funerarias en Fresno de Río Tirón (Burgos)», Veleia, e.p.

GORROCHATEGUI, J. y FERNÁNDEZ CORRAL, M., 2015: «Cinco nuevas estelas funerarias procedentes de Belorado (Burgos)», Zeitschrift für Papyrologie und Epigraphik, e.p.

GONZÁLEZ RODRÍGUEZ, M. C., 1986: Las unidades organizativas indigenas del área indoeuropea de Hispania, Vitoria-Gasteiz.

(Abásolo Álvarez, Albertos Firmat y Elorza Guinea, 1975). Sobre otros conjuntos de características propias u officinae ver Mayer, 2012 y Abascal Palazón, 2014. Igualmente, en general sobre las officinae ver Edmondson, 2015. 
GONZÁLEZ RODRÍGUEZ, M. C., 1994: «Las unidades organizativas indígenas II: Addenda et corrigenda», $\mathrm{Ve}$ leia, 11, 169-176.

GONZÁLEZ RODRÍGUEZ, M. C., 1997: Los astures y los cántabros vadinienses: problemas y perspectivas de análisis de las sociedades indígenas de la Hispania indoeuropea, Vitoria-Gasteiz.

GONZÁLEZ RODRÍGUEZ, M. C., 2002: «Epigrafía y sociedad: el ejemplo vadiniense», en J. A. Solórzano Telechea y M. R. González Morales (eds.), Actas del II Encuentro de Historia de Cantabria (Santander 2002), 91-115, Santander.

GONZÁLEZ RODRÍGUEZ, M. C., y RAMÍREZ SÁNCHEZ, M., 2011: «Unidades organizativas indigenas del área indoeuropea de Hispania III: Addenda», Veleia, 28, 253-268.

MAURIN, L. y KHANOUSSI, M., 2003: Mourir à Dougga. Recueil des inscriptions funéraires, Bordeaux-Túnez.

MAYER, M., 2012: «El material lapídeo como elemento identificativo de officinae epigráficas», en A. Donati y G. Poma (eds.), L'officina epigrafica romana. In ricordo di Giancarlo Susini, 89-107, Faenza.

OLLER, J., DE LEÓN, D., y EGUILUZ, D., 2015: Informe de la intervención arqueológica en los yacimientos de La Muela, La Mesa y El Castillo. Campaña 2014, (Informe inédito).

RAMÍREZ SÁNCHEZ, M., 1999: Epigrafía y organización social en la región celtibérica: los grupos de parentesco, Universidad de Las Palmas de Gran Canaria, Tesis doctoral inédita.
RAMÍREZ SÁNCHEZ, M., 2003: «Epigrafía latina y relaciones de parentesco en la región celtibérica. Nuevas propuestas», en S. Armani, B. Hurlet-Martineau y A. U.Stylow (eds.), Epigrafía y sociedad en Hispania durante el Alto Imperio: estructuras y relaciones sociales, 13-31, Alcalá de Henares.

RAMÍREZ SÁNCHEZ, M., 2004: «Estelas funerarias y grupos de parentesco en la región celtibérica», en Actas del VII Congreso Internacional de Estelas Funerarias, 139-156, Santander.

RAMÍREZ SÁNCHEZ, M., 2007: «Los grupos de parentesco en la epigrafía hispánica: genitivos de plural en -on/om», en A. G. M. Mayer y G. Baratta (eds.), Actas del XII Congressus Internationalis Epigraphiae Graecae et Latinae, 1161-1168, Barcelona.

REYES HERNANDO, O. V., 2000: El Conjunto epigráfico de Belorado, Valladolid.

RIB I = COLLINGWOOD, R. G. y WRIGHT, R. P., 1965: The Roman Inscriptions of Britain I, Stroud, Gloucestershire.

RUIZ DE HUIDOBRO, L., 1950: Las peregrinaciones jacobeas, Madrid.

SALLER, R. P., y SHAW, B. D., 1984: «Tombstones and Roman Family Relations in the Principate: Civilians, Soldiers and Slaves», The Journal of Roman Studies, 74, 124-156.

SCHEIDEL, W. (ed.), 2001: Debating Roman Demography, Leiden-Boston-Köln.

VALLEJO, J. M., 2005: Antroponimia indigena de la Lusitania romana, Vitoria-Gasteiz.

Recepción: 21-04-2015

Aceptación: 13-07-2015 